\title{
Research on the Simulation Application of Data Mining in Urban Spatial Structure
}

\author{
Jun Zhang, Xin Sui, and Xiong He $\mathbb{D}$ \\ School of Architecture and Urban Planning, Yunnan University, Kunming, Yunnan, China \\ Correspondence should be addressed to Xiong He; ydxh@mail.ynu.edu.cn
}

Received 17 May 2020; Revised 27 June 2020; Accepted 21 July 2020; Published 3 August 2020

Academic Editor: Naixue Xiong

Copyright (c) 2020 Jun Zhang et al. This is an open access article distributed under the Creative Commons Attribution License, which permits unrestricted use, distribution, and reproduction in any medium, provided the original work is properly cited.

\begin{abstract}
Data mining and simulation of the Internet of things (IOT) have been applied more and more widely in the rapidly developing urban research discipline. Urban spatial structure is an important field that needs to be explored in the sustainable urban development, while data mining is relatively rare in the research of urban spatial structure. In this study, 705,747 POI (Point of Interest) were used to conduct simulation analysis of western cities in China by mining the data of online maps. Through kernel density analysis and spatial correlation index, the distribution and aggregation characteristics of different types of POI data in urban space were analyzed and the spatial analysis and correlation characteristics among different functional centers of the city were obtained. The spatial structure of the city is characterized by "multicenters and multigroups", and the distribution of multicenters is also shown in cities with different functional types. The development degree of different urban centers varies significantly, but most of them are still in their infancy. Data mining of Internet of things (IOT) has good adaptability in city simulation and will play an important role in urban research in the future.
\end{abstract}

\section{Introduction}

The concept of sustainable development was put forward in 1987, with the speeding up of globalization and urbanization; China's urban economy entered a period of rapid growth, the speed of urban development is accelerating and the scale of urban construction is generally increasing. However, at the same time, the urban problems brought about by the rapid development period have also attracted the attention of urban researchers. In the view of many scholars, the ideal sustainable urban spatial structure can alleviate or solve the problems that arise in the process of urban development in a limited way, which is an innovation of its mode and an unremitting exploration of the ideal urban spatial structure.

The development of the city presents a feature of continuous diffusion and reaggregation in space. In the process of aggregation and expansion in urban development, a gathering center will be formed in space. The gathering center is the space carrier of urban public activities and the core area of urban activities [1]. With the phenomenon of diffusion and aggregation more obvious, the urban spatial structure has evolved from the original single center to a multicenter spatial structure $[2,3]$. The multicenter spatial structure has quickly become a research hotspot and research difficulty in urban development research. Under the guidance of the current national macro policy and deliberate guidance of planners, megacities such as Beijing, Shanghai, Guangzhou, and Shenzhen have begun to use multicenters as the core goal of urban space development strategy [4-6].

Chinese scholars' research on the spatial structure of urban centers mainly involves urban center theory research $[7,8]$, central structure measurement and efficiency research [9-12], performance evaluation [8,13-15], organization and governance $[16,17]$, etc. Most of China's domestic analysis is based on geospatial analysis and morphological analysis. The urban central structure is analyzed from the distribution characteristics of different elements of urban space [18, 19]. However, there are few studies on the spatial structure of urban centers from different functional types.

In recent years, the use of urban mass data to analyze the spatial structure of cities has provided a new research 
paradigm for the sustainable development of cities. The use of a wider range of urban data includes rail transit card data, mobile phone signaling data, network review data, thermal map data, microblogging sign-in data, and night lighting data. Ying, Feng, Zhiqiang, Yang, and Xiong analyzed the spatial structure of the city through these "big data" cities [20-25]. However, based on the big data of these cities, there are problems in data acquisition difficulties, fewer ways, and inability to update in real time. Based on the open source data POI (Point of Interest), it provides an accurate and effective alternative to urban research. The POI data is an expression of a virtual abstraction of a real geographical entity in space, has spatial attribute information, has a large amount of data, and is easy to acquire. It is one of the most important data in the study of urban geography. It has been widely used in urban research. Chinese scholars' research on POI data mainly focuses on urban spatial structure research [26], urban functional area identification [27], etc. Based on POI data, urban spatial structure can be well recognized, but through geographic information system and POI big data to cities. There is still little research on the development of the central space structure.

At present, the research on a large number of urban center spatial structures is mainly concentrated in first-tier cities and megacities such as Beijing, Shanghai, and Guangzhou. There is less attention to general provincial capital cities. Choosing China's Guiyang as a research object is more representative. Guiyang is one of the core cities in western China and one of the birthplaces of China's "big data." In Guiyang's urban sustainable development strategy, the development concept of building a multicenter city is also mentioned, and the spatial structure layout of the city's multicenter is formed. To this end, this study uses the POI data of Guiyang City from 2016 to 2018, taking the main city of Guiyang as an example and using the nuclear density analysis and spatial correlation index in geography to analyze the evolution characteristics of urban centers in the main urban area of Guiyang. As one of the central cities in the west, Guiyang analyzes the spatial structure of the development of Guiyang multicenter. Through the analysis of Guiyang, it summarizes the achievements and shortcomings of the development of Guiyang urban center, and it provides new thinking for city planning and sustainable development mode of the city through the development of Guiyang urban center and the optimization of spatial structure.

Big data are used to simulate the urban spatial structure to achieve the purpose of exploring the urban spatial form of Guiyang, the capital city of western China. And further explore the sustainable development model of urban spatial structure.

\section{Methodology}

2.1. Study Area. Guiyang is the capital city of Guizhou province in western China and the central city in western China. By 2018, Guiyang had a permanent resident population of 3.136 million, with a GDP of US $\$ 53.672$ billion, ranking the second fastest growth rate in China. Since March 2015, cities in western China have been developing slowly due to their inland location. The National Development and Reform Commission and other departments jointly issued the "One Belt And One Road" development strategy, bringing historical development opportunities to western cities. The urban spatial structure of Guiyang has undergone drastic changes (Figure 1).

2.2. Data Source. The data for this study was from December 2016 to December 2018. Get online map POI data about Guiyang, China, through an open interface. POI data has been widely used in urban navigation systems in urban geography, with spatial attributes and location information of urban entity objects. Therefore, the POI data basically contains all the entity objects in the study area urban space. As a kind of big data of geographic information, POI is widely used in the simulation of geographic space, especially in urban space, due to its advantages such as large quantity, fast update, and accurate positioning. Compared with other big data in model cities, POI has a better result.

After obtaining the POI data (http://www.amap.com), the data is verified and cleaned up. Among them, the 2016 POI data is 204,000, the 2017 POI data is 248516, and the 2018 POI data is 253231, a total of 705747. According to the online map POI classification system combined with different functional attributes of the city, 705,747 POI data are divided into five categories: public service, life service, leisure and entertainment, residence, and business (Table 1).

2.2.1. Kernel Density Estimation [28]. Kernel density analysis simulates the distribution of density by calculating the density values of points in the space and the neighborhood of the line features. In recent years, nuclear density analysis has been widely used in the study of spatial distribution of geography. This study compares the results of each nuclear density analysis under different search radii to explore the spatial distribution of the overall urban and urban areas of Guiyang's main urban area and different types of POI:

$$
p_{i}=\frac{1}{n \pi R^{2}} \times \sum_{j=1}^{n} k_{j}\left(1-\frac{D_{i j}^{2}}{R^{2}}\right)^{2},
$$

where $k_{j}$ is the spatial weight of the research object $j$ (POI); $D_{i j}$ is the distance between different POIs in space; and $R$ is the bandwidth within the search area (bandwidth).

2.2.2. Spatial Correlation Index [29]. The spatial correlation index is currently used to describe the characteristics of cities in geospatial. This study uses the Getis-Ord General $G$ and Getis-Ord $\mathrm{Gi}^{*}$ indices to measure the global and local features, structural patterns, and clustering of spatial locations in urban geospatial space, respectively. The spatial distribution of hot spots and cold spots is used to indicate the degree of association.

Getis-Ord General G:

$$
G(d)=\frac{\sum \sum w_{i j}(d) x_{i} x_{j}}{\sum \sum x_{i} x_{j}}
$$




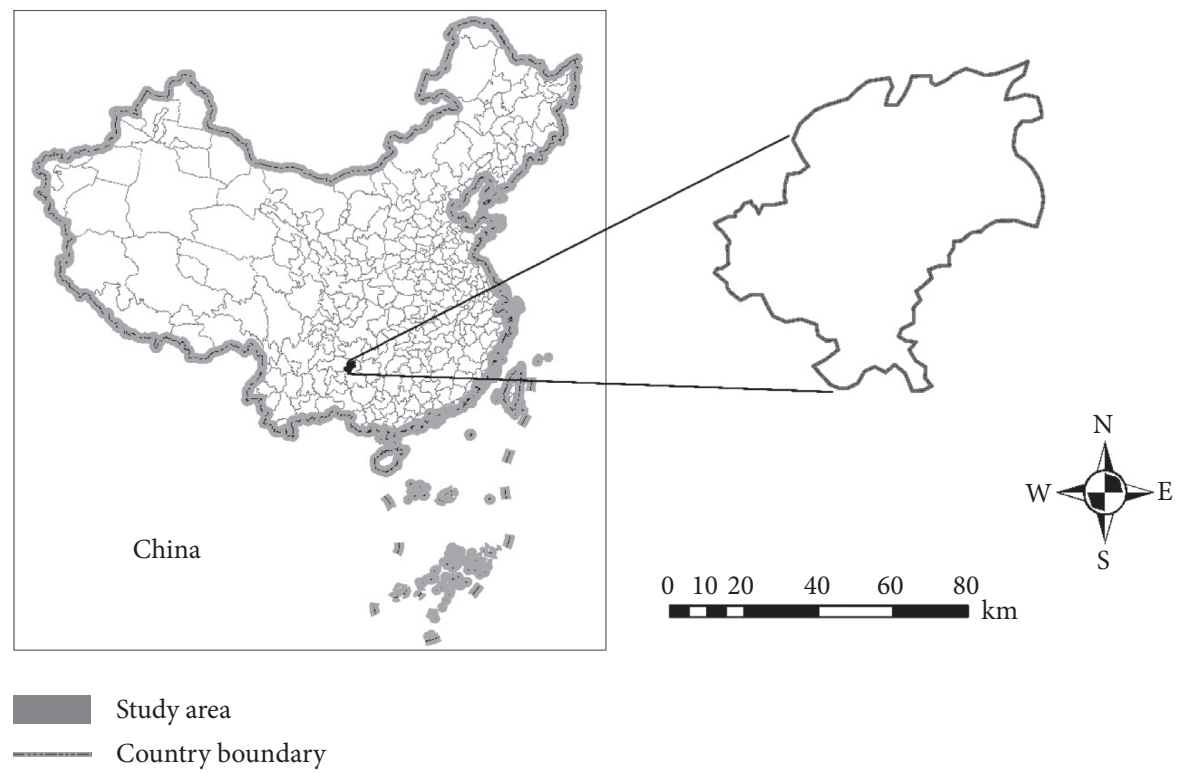

FIgURE 1: Study area.

TABle 1: Classification of urban POI data in Guiyang.

\begin{tabular}{lcc}
\hline POI classification & Classified content & Quantity (\%) \\
\hline Public service & Road auxiliary facilities, public facilities, transportation & \\
& facilities services, access facilities, government agencies and & \\
Domestic services & social groups, and cultural and educational services & $132117 / 18.72$ \\
Leisure and entertainment & Catering services, shopping services, and health-care services & $435803 / 61.75$ \\
$\begin{array}{l}\text { Reside } \\
\text { Busines }\end{array}$ & Resorts, golf-related, entertainment venues, and sports venues & $49442 / 7$ \\
Total & Community, villa area, and dormitory & $26031 / 3.68$ \\
\hline
\end{tabular}

where $d$ is the distance of Guiyang city center; $w_{i j}(d)$ is the spatial weight in the study area; and $x_{i}$ and $x_{j}$ are the extended intensity index of urban land. Under the assumption that space does not agglomerate, the expected value of $G(d)$ is $E(G)$ :

$$
\begin{aligned}
E(G) & =\frac{W}{[n(n-1)]}, \\
w & =\sum \sum w_{i j}(d) .
\end{aligned}
$$

Under the condition of positive distribution, the statistical test value of $G(d)$ is $Z(G)$ :

$$
\begin{gathered}
Z(G)=\frac{[G-E(G)]}{\sqrt{\operatorname{Var}(G)}} \\
{\left[E(G)=W_{n}(n-1)\right] .}
\end{gathered}
$$

When $G(d)$ is higher than $E(G)$ and the $Z(G)$ value is significant, high-value clusters appear in the study area; when $G(d)$ approaches $E(G)$, the study area variables appear randomly distributed:

$$
Z\left(G_{i}^{*}\right)=\frac{\sum_{j}^{n} w_{i j}(d) x_{j}}{\sum_{j}^{n} x_{j}} .
$$

\section{Result}

\subsection{Urban Center Evolution Process Based on Nuclear Density Analysis}

3.1.1. Evolution of the Overall Urban Center. In nuclear density analysis, different search radii will result in different results for density analysis. The search radius of 500, 1000, 1500 , and $2000 \mathrm{~m}$ was set to compare the density of POI in Guiyang main city from 2016 to 2018. It is found that, as the search radius increases, the internal POI will gradually merge. When the search radius is increased to a certain distance, the spatial agglomeration characteristics of the POI will be weakened to some extent. In general, when the search radius is small, a small range of POI aggregation areas can be identified. When the search radius is increased, it can reflect the macroscopic scale of the urban spatial structure, and the nuclear density equivalent curve will be smoother. This study explores the evolution of the urban center structure and 
urban center. According to the scale comparison of existing urban research and the overall and local effects of different search distances in Kunming, $1500 \mathrm{~m}$ is finally selected as the nuclear density search radius of this study.

Based on the search radius $R=1500 \mathrm{~m}$, the nuclear density analysis of Guiyang three-year POI was carried out. It can be seen from Figure 2 that, in 2016, Guiyang City Center is Times Square, Longjiyuan and Tianjiao Haoyuan are subcenters, and Sunshine Garden and Ganjing Bridge have large groups. There are also obvious groups on both sides of Guanshan Lake. In 2017, Guiyang City Center was Times Square, but the city's subcenter changed, with Sunshine Garden surpassing Tianjiao Haoyuan and Longjiyuan as the city's subcenter. The group on both sides of the Ganjing Bridge and Guanshan Lake has an increasing trend, and a new urban group is formed in Longwan International. In 2018, the city center is still the Times Square. The subcenter of the city has undergone significant changes. The Longwan International Group has the fastest development, and the concentration is close to Longjiyuan, becoming a new subcenter of the city. The concentration of urban groups on both sides of the Ganjing Bridge and Guanshan Lake continues to increase (Figure 2).

Analysis of the POI nuclear density map of Guiyang City from 2016 to 2018, we can find that Guiyang has begun to have a multicenter spatial structure in the city since 2016. By 2018, the urban multicenter spatial structure has become more apparent. The main city of Guiyang is dominated by Times Square and has the widest range of radiation. It starts from Yunyan District in the north and goes to Guiyang Station in the south. In 2016-2018, there was a significant change in the city center of Guiyang. The main center of Guiyang City has been growing around Times Square. And it is very obvious to expand to Shantou Park. Guiyang City subcenter has a clear growth trend, but Tianjiao Garden is not growing faster than Sunshine Garden and Longwan International Group. There are many agglomeration groups in the main city of Guiyang, among which the development of Longwan International and Sunshine Garden Group is the most significant. The development of the groups on both sides of Guanshan Lake is slow, and the Ganshan Bridge also has obvious growth, but there is still a big gap from the city's subcenter.

Guiyang, China, is already developing into a multicenter city, but the development of the main and secondary centers is unbalanced. The growth of the main center in Guiyang is more obvious and the development is relatively perfect, but there is still a large space for the development of the subcenter and each group. In addition to the main center, the other secondary centers show the characteristics of dynamic growth. In general, the development of the spatial structure of the Guiyang urban center has initially highlighted the characteristics of dynamic "multicenter" development.

3.1.2. Evolution Process of Different Types of Urban Centers. Due to the urban development, planning policy guidance, and the public role of natural conditions, there are significant differences in the spatial distribution and agglomeration of urban centers with different functions. Therefore, different types of POIs tend to have large differences in spatial distribution structures. The nuclear distribution of the centers of different functions is explored by performing nuclear density analysis on the distribution of different types of POIs.

As can be seen from Figure 3, the spatial distribution of different types of POIs is highly variable. In 2016, the public service category POI was centered on Times Square, and there were small groups in Guanji Lake Longjiyuan and Sunshine Garden. The residential POI is centered on Times Square, with Guiyang Station as the subcenter, and the main and subcenters have a tendency to merge with each other. There is a large group presence in Longwan International. The business category POI is centered on Times Square, and a small number of groups exist near Guanshan Lake. The life-oriented POI is centered on Times Square and spreads to the vicinity of Guiyang Station. Ganjing Bridge and Longjiyuan in Guanshanhu District are subcenters. Longwan International, Sunshine Garden, and Tianjiao Haoyuan have large urban groups. Leisure POI is centered on Times Square, and Longwan International is a group.

In 2017, the public service category POI was centered on Times Square, and the Longwan International Group had a growing trend. The residential POI is centered on Times Square and the subcenter has been integrated. And they grew to Guiyang Station and Shenlong Cave, respectively. The Guanji Lake Longjiyuan Group continued to grow. The business category POI is centered on Times Square and Shantou Park is the subcenter. Longjiyuan has a group presence, which is a big change from 2016. The life class POI is centered on Times Square. The radiation area has further increased, and the concentration of Longwan International, Sunshine Garden, and Tianjiao Haoyuan City has caught up with the Ganjing Bridge and Longjiyuan in Guanshanhu District. The same as the city's subcenter, a new city group appeared on both sides of Guanshan Lake. The concentration of leisure POI urban main centers and urban groups has further increased.

In 2018, the public service POI was centered on Times Square, and the growth trend of the main center was more obvious than the growth trend of the group. The residential POI is centered on Times Square, and the growth trend toward Guiyang Station and Shenlong Cave is more obvious. The business category POI is centered on Times Square, and the Shantou Park subcenter tends to merge with the main center. In the vicinity of Guanshanhu Park, the phenomenon of urban group formation is more obvious. The life class POI is centered on Times Square. A new subcenter was formed near Guiyang Station, and the concentration of POI in the other subcenters was also more obvious. The leisure POI city main center has a differentiation trend in the direction of Shenlong Cave.

From the analysis of different types of POI nuclear density maps from 2016 to 2018, we can find that, in the past three years, different types of POIs are based on Times Square. The development of urban centers with different functions is quite different. Among them, the development of living services and residential urban centers is relatively perfect, but the center of life services is growing faster than leisure centers. 


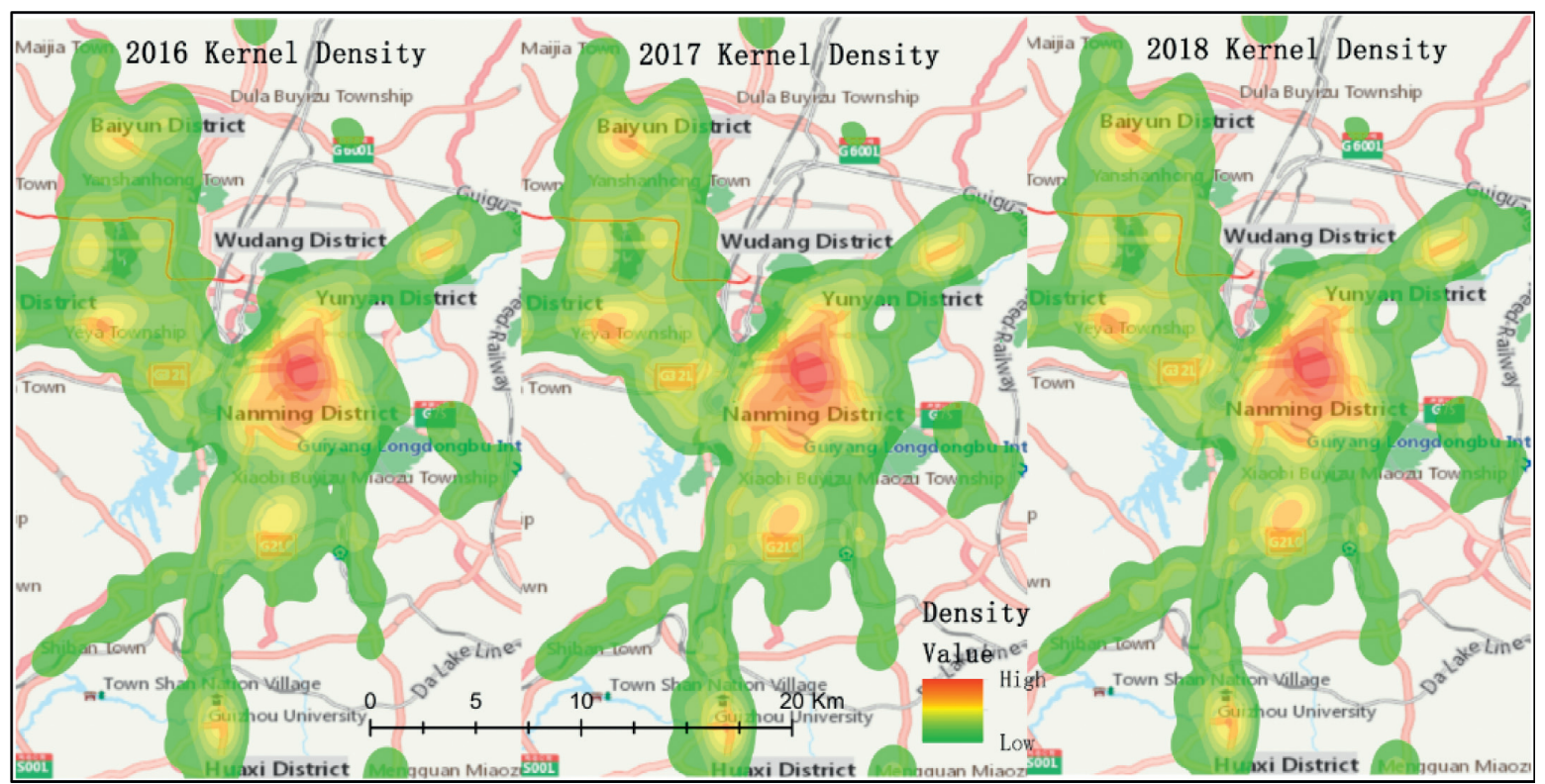

FIgURe 2: POI nuclear density from 2016 to 2018.

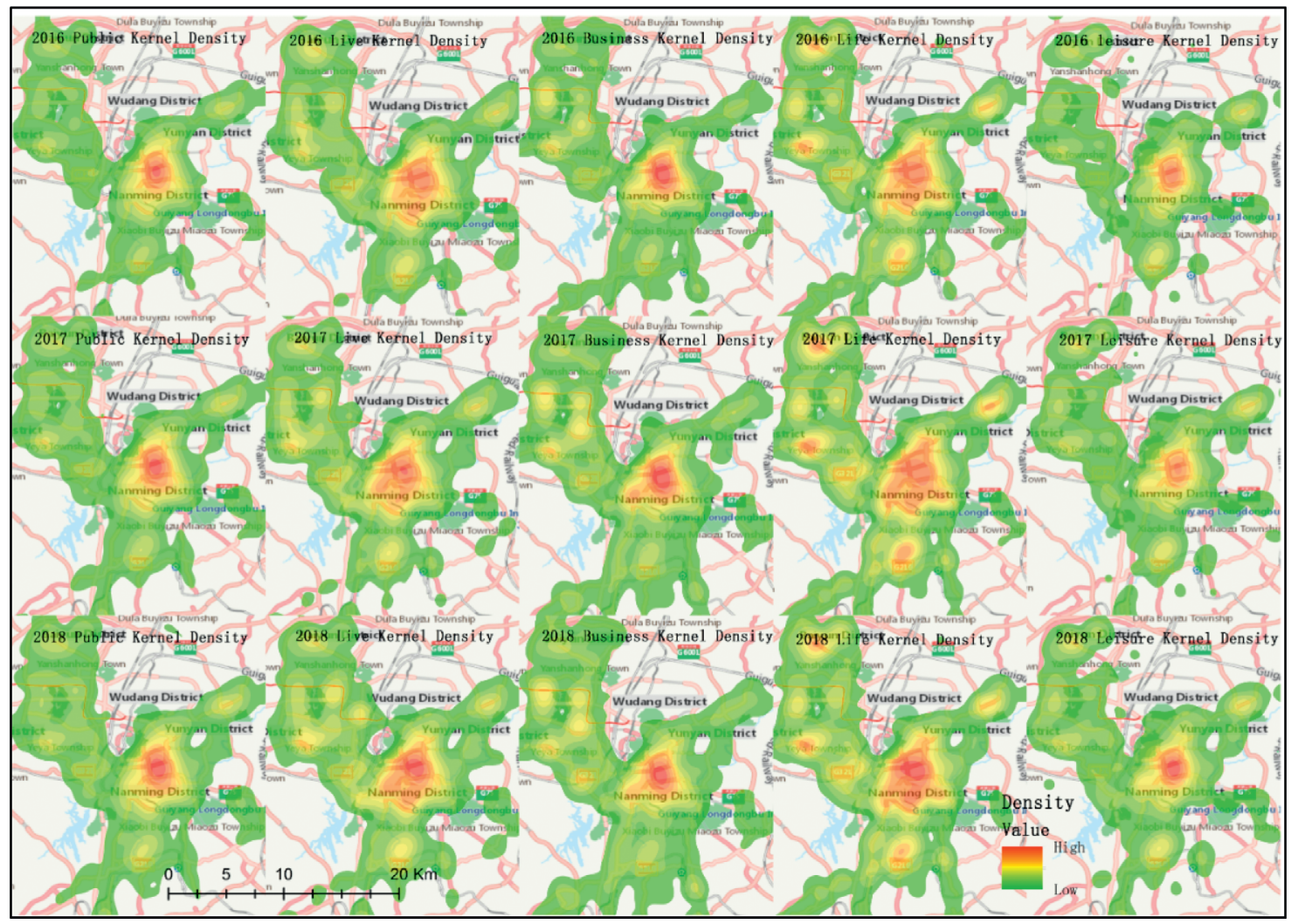

Figure 3: Nuclear density of different types of POIs from 2016 to 2018.

The public service and business city centers are generally developed. The growth rate of the main center is much larger than that of the subcenters and groups. Leisure basically exists in the form of a single center.

There are great imbalances in the development of different functional city centers in Guiyang, China. Some urban centers only have one single center, while the degree of development of the main centers in other cities is much higher than that of the secondary centers and clusters. There is even a situation that the secondary centers and clusters are assimilated into the main centers with the development of the main centers. The multicenter of urban function is still in its infancy, and the spatial structure of urban center also presents an obvious dynamic change. 
3.2. Urban Center Evolution Based on Spatial Correlation Index. Based on the evaluation unit of Guiyang City and the degree of urban POI aggregation, the spatial growth correlation index of the city center of Kunming, Getis-Ord General $G$ and Getis-Ord Gi*, is calculated. Thus, the global and local characteristics of the city center due to spatial correlation are described.

The global characteristics are shown in Table 2: in three years, the observation value $G(d)$ of the global statistical indicators of the Guiyang City Center Growth Correlation Index is greater than the expected value $E(d)$, and the $Z$ score is obvious, indicating that it is in the main city of Guiyang. There is a phenomenon of high/low value agglomeration, but the $Z$ value rises and falls, and the agglomeration phenomenon also increases and decreases. The overall $Z$ value is the highest in 2016-2018, indicating that the growth of POI in Guiyang has been growing around a few cores in the past three years. Except for the overall $Z$ value, the $Z$ values of other functional POIs have increased, and the central polarization has been strengthened; 2016-2018 The public service, living service, and business $Z$ value are relatively high, indicating that the growth of three types of POIs around several centers in three years is obvious; in 2016-2018, the $Z$ value of public services and life services has increased rapidly, and the central polarization has strengthened. And the $Z$ value of life service rose the fastest, indicating that the polarization intensity of the high/low agglomeration center is rapidly increasing. The above analysis also verified the results of the nuclear density analysis.

The spatial correlation index Getis-Ord Gi* in the threeyear evaluation unit is calculated separately, and the highlevel cartographic representation based on ArcGIS is used to obtain the "hot spot area" and "cold spot area" distribution map of the POI growth intensity in the main urban area of Guiyang (Figure 4). The hot spot area and the "cold spot area" have obvious evolution and migration processes in the spatial distribution.

Analysis of the distribution map shows that, from 2016 to 2018, with the acceleration of the urbanization process in Guiyang, the urban POI increased, the number of "hot spots" increased significantly, and the "cold spot area" decreased continuously. It has risen from $33.6 \%$ in 2016 to $38.2 \%$ in 2017 and reached a maximum of $40.8 \%$ in 2018 . In the three periods, the "cold spot zone" became a significant downward trend, accounting for $33.9 \%, 29.7 \%$, and $24.8 \%$, respectively. In the three periods, except for Times Square, which has been in the "hot spot", "hot spot" such as Longwan International, Longjiyuan, and Sunshine Garden has been constantly evolving and changing.

In 2016-2018, there was no obvious change in the "hot spot" of public service category, mainly due to the increase in the number of hotspots and cold spots, and the growth of the hotspots as Times Square, but there were also new hotspots in Longwan International.

In 2016-2018, the "hot spot" of the residential category changed more obviously. First of all, the "hot spot" increased to $69.7 \%$ within three years. Secondly, outside the Times Square, new growth cores appeared in Longwan International, Longjiyuan, and Sunshine Garden. The growth nuclear periphery was followed by high-value area and median area and low-value area; the peripheral structure is more obvious.

In 2016-2018, the change of "hot spot" in business category mainly showed that it changed from south of Times Square to the north, and more "cold spot areas" were converted into median areas, but the increase of "hot spots" was not obvious.

The "hot spot" of life service category has the most significant changes. First, the "cold spot area" is all converted into "hot spot area" and "media area", and then the "hot spot area" is spread to the whole city, in Times Square, Longwan International, Guanjihu District Longjiyuan, and Sunshine Garden have all become the core of growth and have reached the development level of urban multicenter.

The leisure "hot spot" has not changed significantly in three years, and its growth rate is slow.

According to the above analysis, in 2016-2018, the growth characteristics of various POIs in Guiyang City showed a rapid growth process, and the "hot spot" was roughly reflected in the development process of the upper and lower ends of Times Square. In 2016-2018, various POIs are mainly concentrated in Times Square, which guides the growth of Guiyang City Center. During the continuous expansion of the "hot spot," it gradually evolved into a relatively continuous gathering area in the Guanshan Lake area, and it tends to develop in the direction of Longjiyuan in Guanshanhu District. In the three-year change, basically the spatial structure of the single center of Times Square has been transformed into a spatial structure led by Times Square, Longwan International, Guanshan Lake, Longjiyuan, and many other city centers.

It can be seen from the spatial correlation index GetisOrd General $G$ and Getis-Ord Gi* that the spatial structure change of the urban center of Guiyang is a very obvious dynamic "multicenter" development process. However, except the main urban centers, the other centers were not significantly developed. With the passage of time, the sustainable development model of the urban center spatial structure in Guiyang is bound to be a dynamic "multicenter" development.

\section{Discussion}

4.1. Simulation of Urban Center Evolution Based on POI Data. This study studied the spatial structure of urban centers based on the analysis of POI data from 2016-2018 through geographic information technology. For the perspective of urban geography research and the research paradigm, the POI can describe in detail the geographical distribution and agglomeration of various elements in the city and then identify the geospatial structure of the city. Compared with traditional conventional data, POI data has better observability and timeliness. The clustering characteristics of POI can quickly reflect the problems of urban geospatial construction, industrial distribution, and functional improvement. Therefore, it provides a strong reference for urban development. However, the POI data is only the abstract expression of the urban entity in geospatial space, lacking the 
Table 2: Getis-Ord General G index of POI in Guiyang main city from 2016 to 2018.

\begin{tabular}{cccccccc}
\hline & & Total & Public service & Domestic services & Leisure & Live & Business \\
\hline \multirow{4}{*}{2016} & $G(d)$ & 0.000087 & 0.000174 & 0.000159 & 0.000180 & 0.000203 & 0.000246 \\
& $E(d)$ & 0.000019 & 0.000077 & 0.000096 & 0.000117 & 0.000155 & 0.000110 \\
& $Z$ & 154.765599 & 49.719755 & 31.666177 & 18.253169 & 18.999336 & 35.250216 \\
\hline \multirow{3}{*}{2017} & $G(d)$ & 0.000078 & 0.000169 & 0.000137 & 0.000165 & 0.000220 & 0.000198 \\
& $E(d)$ & 0.000018 & 0.000079 & 0.000080 & 0.000114 & 0.000174 & 0.000089 \\
& $Z$ & 154.646603 & 54.024563 & 36.362221 & 17.605138 & 18.541761 & 41.195000 \\
\hline \multirow{2}{*}{2018} & $G(d)$ & 0.000072 & 0.000133 & 0.000004 & 0.000165 & 0.000214 & 0.000174 \\
& $E(d)$ & 0.000018 & 0.000055 & 0.000002 & 0.000104 & 0.000165 & 0.000085 \\
& $Z$ & 156.753601 & 70.542463 & 146.959363 & 22.548152 & 20.214073 & 41.786830 \\
\hline
\end{tabular}

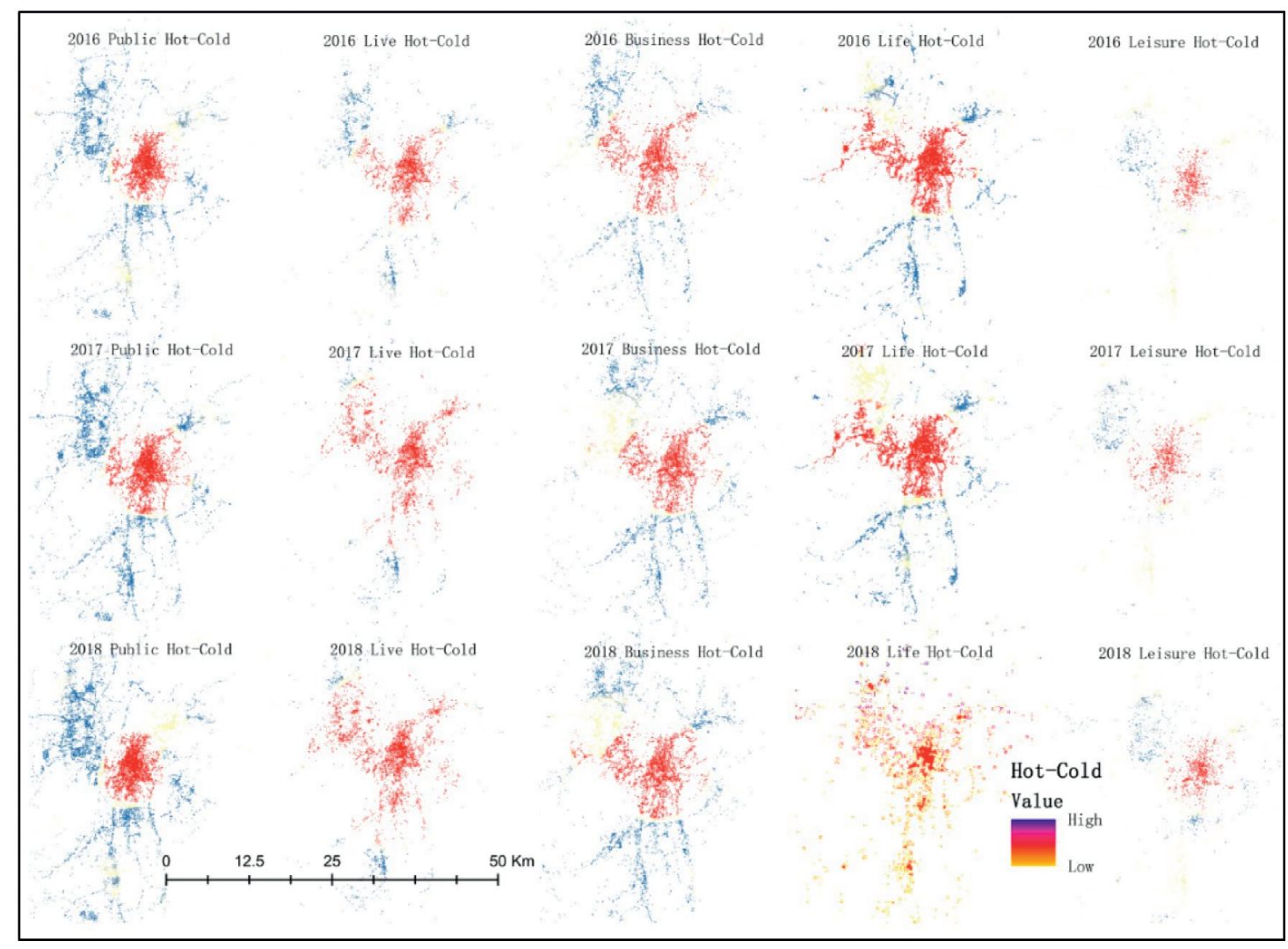

Figure 4: The evolution of the "hot spot" in the main urban area of Guiyang from 2016 to 2018.

detailed information of the attributes of the geographic entity. This study does not consider the grade and scope of the POI in the urban space. In the future, the spatial weight of the POI will be deeply studied.

\subsection{The Wide Application of Big Data to Simulate Urban} Space. It can be seen from this study that big data has been playing an increasingly important role in urban spatial computing. In today's development of new disciplines, traditional data in urban simulation computing can no longer adapt to the development of new disciplines. More and more big data should be introduced into urban simulation calculation, among which POI data is a typical one. POI big data has been used to simulate the calculation of cities with good results. In future studies, more and more updated big data should be introduced to calculate cities in order to obtain better results.

4.3. Prospects for the Integration of Geographic Information Technology and Urban Geographic Big Data. The spatial structure of urban centers based on sustainable development belongs to the discussion of the sustainability of urban space. The research on the sustainability of urban space is a hot issue in urban development, and it is also an urgent problem needed to be solved. The sustainable development model of urban spatial structure is closely related to urban nature, urban population, land use scale, infrastructure, and other factors. This study studied the spatial structure distribution of urban centers through the analysis of urban big data and analyzed that the sustainable development mode of Guiyang 
urban space is a dynamic "multicenter" development, which provides a good reference for the sustainable development of urban space structure in terms of exploration methods and results.

\section{Conclusion}

This study is based on POI data, using nuclear density analysis and spatial correlation index, according to the distribution, agglomeration, and correlation characteristics of POI data in space. The evolution characteristics of different urban centers in the main urban area of Guiyang City were discussed, and the following preliminary conclusions were obtained.

Guiyang's main urban area presents a more obvious "multicenter, multigroup" space structure, and Times Square dominates Guiyang's urban development.

Under five different types of functions, the urban structure has a relatively obvious multicenter distribution. Public services, living services, residence, and business functions are relatively complete. The public service and life service center are mature. In particular, the Life Service Center is basically distributed in multiple centers.

In general, the development of the central areas of Guiyang's main city is relatively perfect. Although the multicenter development of the city is still in its infancy, the differences between the centers are obvious, and there is a big gap from the multicenters of the city. However, the centers in Guiyang are independent of each other and have great development prospects. In the future, the spatial structure of urban center in Guiyang will inevitably present a sustainable development model of dynamic "multicenter" development.

The urban spatial structure and form explored through big data is more authentic and objective in the expression of cities. It plays a guiding and leading role in the future urban planning and construction and provides references for urban builders and planners.

\section{Data Availability}

All raw data in this study are available free of charge. Readers who wish to repeat this study can do so through the following link (DOI 10.17605/OSF.IO/MP29E).

\section{Conflicts of Interest}

The authors declare no conflicts of interest.

\section{Authors' Contributions}

Z. J. conducted a theoretical analysis and contributed to drafting the first draft and writing. X. H. improved the experiment and argumentation and scientifically demonstrated the results of the experiment. X. S. has carried out a lot of work improvement on the writing of the article and participated in the experiment.

\section{References}

[1] J. Yang, Z. Biao, and Y. Shi, "On theoretical frameworks of urban center system development," Urban Planning Forum, vol. 1, pp. 33-39, 2012.

[2] S. W. Sun, "City center and city public space: a planning review on the construction of Lujiazui area of Pudong district, Shanghai," City Planning Review, vol. 8, pp. 66-74, 2006.

[3] X. W. Zheng, "Identification and optimization of xi' an urban center system based on open data," Planners, vol. 31, pp. 57-64, 2017.

[4] L. N. Gao, "Study on the relationship between polycentric development and economic performance of urban agglomeration-a case study of Yangtze river delta mega-city region," Science \& Technology Progress and Policy, vol. 35, pp. 46-52, 2018.

[5] J. X. Nie, Y. P. Huang, and Z. R. Shan, "Characteristics and formation mechanism of the urban system of Wuhan metropolitan area: a study based on the perspective of urban network," Modern Urban Research, vol. 12, pp. 110-116, 2018.

[6] F. Q. Li, M. Zhao, M. D. Wu, and J. Z. Huang, "Polycentric mage-city and its mechanism of spatial performance: findings from xiamen based on LBS and census data," Urban Planning Forum, vol. 5, pp. 21-32, 2017.

[7] Z. D. Luo and C. S. Zhu, "Understanding polycentricity by configuration, function and governance," Urban Planning International, vol. 23, pp. 85-88, 2008.

[8] Y. P. Wei and M. Zhao, "Spatial structure and performance of metropolis: interpretation and application of polycentric structure," City Planning Review, vol. 4, pp. 9-16, 2006.

[9] B. D. Sun, T. Tu, W. Shi, and Y. L. Gao, "Test on the performance of polycentric spatial structure as a measure of congestion reduction in megacities: the case study of Shanghai," Urban Planning Forum, vol. 2, pp. 63-69, 2013.

[10] C. R. Ding, "The impact of urban spatial structure and land use pattern on urban transportation," Urban Transport of China, vol. 8, pp. 28-35, 2010.

[11] Y. L. Guo, Study on the Influence of Multi-Center Space Structure on Urban Traffic. Master's Degree, East China Normal University, Shanghai, China, 2011.

[12] K. Yang, "Population distribution and multicenter measurement of great Beijing," China Population Resources and Environment, vol. 25, pp. 83-89, 2015.

[13] F. Q. Li and M. Zhao, "A discussion on housing development in multi-center cities: phenomenon \& planning implications of Chongqing," Urban Planning Forum, vol. 3, pp. 8-19, 2011.

[14] X. H. Wang and B. D. Sun, "The economic performance of the polycentric spatial structure of mega-cities: based on the models of urban economics," Urban Planning Forum, vol. 22, pp. 20-27, 2011.

[15] H. Yan and B. D. Sun, "The impact of polycentric urban spatial structure on energy consumption: empirical study on the prefecture-level and above cities in China," Urban Development Studies, vol. 22, pp. 13-19, 2015.

[16] A. G. Liu and K. Z. Yang, "Comments on Krugman's edge city model," Scientia Geographica Sinica, vol. 21, pp. 315-322, 2001.

[17] J. X. Zhang, X. L. Luo, and J. Yin, "Polycentric mega-city regions and multi-level governance of the yangtze river delta," Urban Planning International, vol. 23, pp. 65-69, 2008.

[18] B. D. Sun and X. H. Wei, "Spatial distribution and structure evolution of employment and population in Shanghai Metropolitan Area," Acta Geographica Sinica, vol. 69, pp. 747758, 2014. 
[19] L. Zhang, W. Z. Yue, and Y. Liu, "Multidimensional analysis of the polycentric urban spatial structure-a case of Hangzhou,” Economic Geography, vol. 37, pp. 67-75, 2017.

[20] Y. Long, Y. Zhang, and C. H. Cui, "Identifying commuting pattern of Beijing using bus smart card data," Acta Geographica Sinica, vol. 67, pp. 1339-1352, 2012.

[21] F. Zhen, B. Wang, and Y. X. Chen, "China's city network characteristics based on social network space: an empirical analysis of sina micro-blog," Acta Geographica Sinica, vol. 67, pp. 1031-1043, 2012.

[22] Z. Q. Wu and Z. N. Ye, "Research on urban spatial structure based on baidu heat map: a case study on the central city of Shanghai," City Planning Review, vol. 40, pp. 33-40, 2016.

[23] J. Cai, B. Huang, and Y. Song, "Using multi-source geospatial big data to identify the structure of polycentric cities," Remote Sensing of Environment, vol. 202, pp. 210-221, 2017.

[24] X. Yang, D. Wang, and F. Jia, "Exploring the disparities in park access through mobile phone data: evidence from Shanghai, China," Landscape and Urban Planning, vol. 181, pp. 80-91, 2019.

[25] X. He, Z. Yang, K. Zhang, P. Yang, and S. Zhang, "The spatial distribution patterns of the catering trade in nanchang based on Internet public reviews," International Journal of Technology, vol. 9, no. 7, pp. 1319-1328, 2018.

[26] B. C. Ding, Y. X. Liu, and G. Chen, "Urban spatial structure of port city in South China sea based on spatial coupling between nighttime light data and POI," Journal of Geo-Information Science, vol. 20, pp. 854-861, 2018.

[27] Z. D. Chen, B. W. Qiao, and J. Zhang, "Identification and spatial interaction of urban functional regions in beijing based on the characteristics of residents' traveling," Journal of GeoInformation Science, vol. 20, pp. 291-301, 2018.

[28] C. Otioma, A. M. Madureira, and J. Martinez, "Spatial analysis of urban digital divide in Kigali, Rwanda," GeoJournal, vol. 84, no. 3, pp. 719-741, 2019.

[29] Y. Wang, X. Li, Y. Kang, W. Chen, M. Zhao, and W. Li, "Analyzing the impact of urbanization quality on $\mathrm{CO} 2$ emissions: what can geographically weighted regression tell us?" Renewable and Sustainable Energy Reviews, vol. 104, pp. 127-136, 2019. 